\title{
The Modified Parseval Equality of Sturm-Liouville Problems with Transmission Conditions
}

\author{
Mudan Bai, Jiong Sun, and Siqin Yao \\ School of Mathematical Sciences, Inner Mongolia University, Hohhot 010021, China \\ Correspondence should be addressed to Jiong Sun; masun@imu.edu.cn
}

Received 5 July 2013; Revised 5 September 2013; Accepted 26 September 2013

Academic Editor: Alberto Cabada

Copyright (C) 2013 Mudan Bai et al. This is an open access article distributed under the Creative Commons Attribution License, which permits unrestricted use, distribution, and reproduction in any medium, provided the original work is properly cited.

We consider the Sturm-Liouville (S-L) problems with very general transmission conditions on a finite interval. Firstly, we obtain the sufficient and necessary condition for $\lambda$ being an eigenvalue of the S-L problems by constructing the fundamental solutions of the problems and prove that the eigenvalues of the S-L problems are bounded below and are countably infinite. Furthermore, the asymptotic formulas of the eigenvalues and eigenfunctions of the S-L problems are obtained. Finally, we derive the eigenfunction expansion for Green's function of the S-L problems with transmission conditions and establish the modified Parseval equality in the associated Hilbert space.

\section{Introduction}

The Sturm-Liouville (S-L) theory, as an active area of research in pure and applied mathematics, plays an important role in solving many problems in mathematical physics and is concerned in many publications [1-7]. It is well known that for the classical S-L problems, the solutions or the derivatives of the solutions are continuous on the interval, but these conditions cannot be satisfied in many practical physical problems. So, a class of S-L operators with "discontinuity," that is, the S-L problems with transmission conditions at an interior point, are concerned by many mathematical and physical researchers [8-10]. Such conditions are known by various names including transmission conditions [11, 12], interface conditions [13-15], jump conditions [16], and discontinuous conditions $[17,18]$.

In this paper, we consider the following Sturm-Liouville equation:

$$
l y:=-y^{\prime \prime}+q(x) y=\lambda y, \quad x \in I=[-1,0) \cup(0,1],
$$

with boundary conditions:

$$
\begin{gathered}
l_{1} y:=\alpha_{1} y(-1)+\alpha_{2} y^{\prime}(-1)=0, \\
l_{2} y:=\beta_{1} y(1)+\beta_{2} y^{\prime}(1)=0,
\end{gathered}
$$

and transmission conditions:

$$
\begin{aligned}
& l_{3} y:=y(0+)-\gamma_{1} y(0-)-\gamma_{2} y^{\prime}(0-)=0, \\
& l_{4} y:=y^{\prime}(0+)-\gamma_{3} y(0-)-\gamma_{4} y^{\prime}(0-)=0,
\end{aligned}
$$

where $\lambda$ is a complex eigenparameter and $q \in L(I, \mathbb{R})$; notice that the potential function $q(x)$ guarantee $y(0 \pm)$ and $y^{\prime}(0 \pm)$ in (4) makes sense (see Theorem 1); all coefficients of the boundary and transmission conditions are real numbers. Throughout this paper, we assume that $\alpha_{1}^{2}+\alpha_{2}^{2} \neq 0, \beta_{1}^{2}+\beta_{2}^{2} \neq 0$, and

$$
\rho=\left|\begin{array}{ll}
\gamma_{1} & \gamma_{2} \\
\gamma_{3} & \gamma_{4}
\end{array}\right|>0 .
$$

We derive the eigenfunction expansion for Green's function of the S-L problem (1)-(4) and establish the modified Parseval equality of the S-L problem with very general transmission conditions at one inner point 0 of the finite interval $[-1,1]$.

The organization of this paper is as follows. After the Introduction, we construct the basic solutions of S-L equation (1) with transmission conditions (4) and obtain the sufficient and necessary condition for $\lambda$ being an eigenvalue of the S-L problem in Section 2. In Section 3, the asymptotic formulas for eigenvalues and eigenfunctions of the S-L problem are 
obtained by using the asymptotic expressions of the solutions, and we prove that the eigenvalues of the S-L problem are bounded below and are countably infinite. Section 4 contains the eigenfunction expansion for Green's function of the S-L problem (1)-(4) and we establish the modified Parseval equality in the associated Hilbert space by using the eigenfunction expansion for Green's function.

\section{The Basic Solutions and Eigenvalues}

We construct the basic solutions of S-L equation (1) with transmission conditions (4) and obtain the sufficient and necessary condition for $\lambda$ being an eigenvalue of the $\mathrm{S}-\mathrm{L}$ problem in this section. At first, we prove the existence of finite limits for all solution $y$ of (1) and its derivative at both sides of 0 point in the following theorem.

Theorem 1. Assume the coefficient function $q(x)$ in $S-L$ equation (1) is real-valued and Lebesgue integrable on $[-1,0)$ and $(0,1]$, that is, $q \in L(I, \mathbb{R})$; then for the solution $y$ of $(1)$, the limits

$$
y(0 \pm)=\lim _{x \rightarrow 0 \pm} y(x), \quad y^{\prime}(0 \pm)=\lim _{x \rightarrow 0 \pm} y^{\prime}(x)
$$

exist and are finite. lemma.

In order to prove Theorem 1, we need the following

Lemma 2 (see [19]). Let $J=(a, b),-\infty \leq a<b \leq \infty$. Let $M_{n, m}(L(a, b), \mathbb{C})$ denote the set of $n \times m$ matrices with entries from $L((a, b), \mathbb{C})(n, m \in \mathbb{N})$.

(1) Suppose that $P, F$ satisfy

$P \in M_{n}(L(a, c), \mathbb{C}) ; \quad F \in M_{n, m}(L(a, c), \mathbb{C})$

for some $c \in(a, b)$, where $M_{n}(L(a, c), \mathbb{C})=M_{n, n}$ $(L(a, c), \mathbb{C})$. For some $u \in J$ and $C \in M_{n, m}(\mathbb{C})$, let $Y$ be the solution of

$$
Y^{\prime}=P Y+F, \quad Y(u)=C .
$$

Then $Y(a)=\lim _{t \rightarrow a+} Y(t)$ exists and is finite.

(2) Suppose that

$$
P \in M_{n}(L(c, b), \mathbb{C}) ; \quad F \in M_{n, m}(L(c, b), \mathbb{C})
$$

for some $c \in(a, b)$. For some $u \in J$ and $C \in$ $M_{n, m}(\mathbb{C})$, let $Y$ be the solution of $(8)$ on $J$. Then, $Y(b)=$ $\lim _{t \rightarrow b-} Y(t)$ exists and is finite.

Proof of Theorem 1. Let $a=0, b=1$, and let $Y=\left(\begin{array}{c}y \\ y^{\prime}\end{array}\right), P=$ $\left(\begin{array}{cc}0 & 1 \\ q-\lambda & 0\end{array}\right)$, and $F=\left(\begin{array}{l}0 \\ 0\end{array}\right)$. Then (1) is equivalent to the equation $Y^{\prime}=P Y+F$ on $(0,1]$. From Lemma 2 , we know that $Y(0+)=$ $\lim _{t \rightarrow 0+} Y(t)$ exists and is finite. This implies that

$$
y(0+)=\lim _{x \rightarrow 0+} y(x), \quad y^{\prime}(0+)=\lim _{x \rightarrow 0+} y^{\prime}(x)
$$

exist and are finite.
Let $a=-1, b=0$. Then, (1) is equivalent to the equation $Y^{\prime}=P Y+F$ on $[-1,0)$. Since $Y(0-)=\lim _{t \rightarrow 0_{-}} Y(t)$ exists and is finite by Lemma 2 , the limits

$$
y(0-)=\lim _{x \rightarrow 0-} y(x), \quad y^{\prime}(0-)=\lim _{x \rightarrow 0-} y^{\prime}(x)
$$

exist and are finite.

Let us define a new inner product in $L^{2}(I)$ as follows, which is associated with transmission conditions (4) and useful to investigate the S-L problem (1)-(4):

$$
\langle f, g\rangle=\rho \int_{-1}^{0} f_{1} \bar{g}_{1} d x+\int_{0}^{1} f_{2} \bar{g}_{2} d x, \quad \text { for } f, g \in L^{2}(I),
$$

where $f_{1}=\left.f(x)\right|_{[-1,0)}, f_{2}=\left.f(x)\right|_{(0,1]}$. It is easy to verify that $\left(L^{2}(I),\langle\cdot, \cdot\rangle\right)$ is a Hilbert space. For simplicity, it is denoted by $H$. The norm induced by the inner product is denoted by $\|\cdot\|_{H}$. We consider the S-L problem (1)-(4) in the associated Hilbert space $H$.

Theorem 3 (see [14]). The S-L problem (1)-(4) is self-adjoint.

We construct two basic solutions $\phi(x, \lambda)$ and $\chi(x, \lambda)$ of $\mathrm{S}$-L equation (1) by the following procedure. At first, we consider the initial-value problem

$$
\begin{gathered}
-y^{\prime \prime}+q(x) y=\lambda y, \quad x \in[-1,0), \\
y(-1)=\alpha_{2}, \quad y^{\prime}(-1)=-\alpha_{1} .
\end{gathered}
$$

By virtue of Theorem 1.5 in [20], the problem (13) has a unique solution $\phi_{1}(x, \lambda)$ for each $\lambda \in \mathbb{C}$, which is an entire function of $\lambda$ for each fixed $x \in[-1,0)$.

Similarly, the initial-value problem

$$
\begin{gathered}
-y^{\prime \prime}+q(x) y=\lambda y, \quad x \in(0,1], \\
y(0+)=\gamma_{1} \phi_{1}(0-, \lambda)+\gamma_{2} \phi_{1}^{\prime}(0-, \lambda), \\
y^{\prime}(0+)=\gamma_{3} \phi_{1}(0-, \lambda)+\gamma_{4} \phi_{1}^{\prime}(0-, \lambda)
\end{gathered}
$$

has a solution $\phi_{2}(x, \lambda)$ for each $\lambda \in \mathbb{C}$. Moreover, $\phi_{2}(x, \lambda)$ is an entire function of $\lambda$ for each fixed $x \in(0,1]$. We define a function $\phi(x, \lambda)$ on $x \in I=[-1,0) \cup(0,1]$ by

$$
\phi(x, \lambda)= \begin{cases}\phi_{1}(x, \lambda), & x \in[-1,0), \\ \phi_{2}(x, \lambda), & x \in(0,1] .\end{cases}
$$

Obviously, $\phi(x, \lambda)$ satisfies S-L equation (1), the boundary condition (2), and both transmission conditions (4); that is, $\phi(x, \lambda)$ is a solution of S-L equation (1).

As same as above, the initial-value problem

$$
\begin{gathered}
-y^{\prime \prime}+q(x) y=\lambda y, \quad x \in(0,1], \\
y(1)=\beta_{2}, \quad y^{\prime}(1)=-\beta_{1}
\end{gathered}
$$


has a solution $\chi_{2}(x, \lambda)$ which is an entire function of $\lambda$ for each fixed $x \in(0,1]$. Similarly, the initial-value problem

$$
\begin{gathered}
-y^{\prime \prime}+q(x) y=\lambda y, \quad x \in[-1,0), \\
y(0-)=\frac{1}{\rho}\left(\gamma_{4} \chi_{2}(0+, \lambda)-\gamma_{2} \chi_{2}^{\prime}(0+, \lambda)\right), \\
y^{\prime}(0-)=-\frac{1}{\rho}\left(\gamma_{3} \chi_{2}(0+, \lambda)-\gamma_{1} \chi_{2}^{\prime}(0+, \lambda)\right)
\end{gathered}
$$

also has a unique solution $\chi_{1}(x, \lambda)$, which is an entire function of $\lambda$ for each fixed $x \in[-1,0)$. Let

$$
\chi(x, \lambda)= \begin{cases}\chi_{1}(x, \lambda), & x \in[-1,0) \\ \chi_{2}(x, \lambda), & x \in(0,1]\end{cases}
$$

By (16) and (17), $\chi(x, \lambda)$ satisfies $\mathrm{S}-\mathrm{L}$ equation (1) and conditions (3) and (4); that is, $\chi(x, \lambda)$ is another solution of S-L equation (1).

It is well known that, from the ordinary linear differential equation theory, the Wronskians $\omega_{j}(\lambda):=W\left(\phi_{j}(x, \lambda)\right.$, $\left.\chi_{j}(x, \lambda)\right)(j=1,2)$ are independent of the variable $x$.

Lemma 4. The equality $\omega_{2}(\lambda)=\rho \omega_{1}(\lambda)$ holds for each $\lambda \in \mathbb{C}$.

Proof. Since the Wronskians $\omega_{j}(\lambda):=W\left(\phi_{j}(x, \lambda), \chi_{j}(x, \lambda)\right)$ $(j=1,2)$ are independent of the variable $x$, then by $(14)$ and (17),

$$
\begin{aligned}
& \omega_{2}(\lambda) \\
& =\left.\omega_{2}(\lambda)\right|_{x=0+} \\
& =\left|\begin{array}{ll}
\phi_{2}(0+, \lambda) & \chi_{2}(0+, \lambda) \\
\phi_{2}^{\prime}(0+, \lambda) & \chi_{2}^{\prime}(0+, \lambda)
\end{array}\right| \\
& =\left|\begin{array}{ll}
\gamma_{1} \phi_{1}(0-, \lambda)+\gamma_{2} \phi_{1}^{\prime}(0-, \lambda) & \gamma_{1} \chi_{1}(0-, \lambda)+\gamma_{2} \chi_{1}^{\prime}(0-, \lambda) \\
\gamma_{3} \phi_{1}(0-, \lambda)+\gamma_{4} \phi_{1}^{\prime}(0-, \lambda) & \gamma_{3} \chi_{1}(0-, \lambda)+\gamma_{4} \chi_{1}^{\prime}(0-, \lambda)
\end{array}\right| \\
& =\rho \omega_{1}(\lambda) .
\end{aligned}
$$

Let $\omega(\lambda)=\omega_{1}(\lambda)$. Then $\omega(\lambda)=(1 / \rho) \omega_{2}(\lambda)$ and is an entire function of $\lambda$.

Theorem 5. The eigenvalues of the S-L problem (1)-(4) coincide with the zeros of the function $\omega(\lambda)$.

Proof. Let $\lambda_{0}$ be an eigenvalue of the S-L problem (1)-(4) and let $y\left(x, \lambda_{0}\right)$ be any corresponding eigenfunction. Let us assume that $\omega\left(\lambda_{0}\right) \neq 0$. Then, $W\left(\phi_{j}\left(x, \lambda_{0}\right), \chi_{j}\left(x, \lambda_{0}\right)\right) \neq 0(j=$ $1,2)$. Consequently, each pair of functions $\phi_{1}\left(x, \lambda_{0}\right), \chi_{1}\left(x, \lambda_{0}\right)$ and $\phi_{2}\left(x, \lambda_{0}\right), \chi_{2}\left(x, \lambda_{0}\right)$ is linearly independent. Therefore, the solution $y\left(x, \lambda_{0}\right)$ of (1) may be represented in the form

$$
y\left(x, \lambda_{0}\right)= \begin{cases}c_{1} \phi_{1}\left(x, \lambda_{0}\right)+c_{2} \chi_{1}\left(x, \lambda_{0}\right), & x \in[-1,0), \\ d_{1} \phi_{2}\left(x, \lambda_{0}\right)+d_{2} \chi_{2}\left(x, \lambda_{0}\right), & x \in(0,1],\end{cases}
$$

where at least one of the constants $c_{1}, c_{2}, d_{1}, d_{2}$ is not zero. Since $y\left(x, \lambda_{0}\right)$ satisfies boundary conditions (2) and (3), we obtain $c_{2}=0, d_{1}=0$. And substituting (20) in condition (4), we get $c_{1}$ and $d_{2}$ satisfying the following equations:

$$
\begin{aligned}
& d_{2} \chi_{2}\left(0+, \lambda_{0}\right)-c_{1}\left(\gamma_{1} \phi_{1}\left(0-, \lambda_{0}\right)+\gamma_{2} \phi_{1}^{\prime}\left(0-, \lambda_{0}\right)\right)=0 \\
& d_{2} \chi_{2}^{\prime}\left(0+, \lambda_{0}\right)-c_{1}\left(\gamma_{3} \phi_{1}\left(0-, \lambda_{0}\right)+\gamma_{4} \phi_{1}^{\prime}\left(0-, \lambda_{0}\right)\right)=0
\end{aligned}
$$

From (14), the determinant of the matrix of coefficients in the last equations is equal to $\omega_{2}\left(\lambda_{0}\right)$ which is not zero by the assumption. Hence, $c_{1}=0$ and $d_{2}=0$. This is a contradiction. Thus, each eigenvalue of the S-L problem (1)-(4) is a zero of the function $\omega(\lambda)$.

Conversely, let $\omega\left(\lambda_{0}\right)=0$. Then, $W\left(\phi_{1}\left(x, \lambda_{0}\right)\right.$, $\left.\chi_{1}\left(x, \lambda_{0}\right)\right)=0$ for all $x \in[-1,0)$. Consequently, the functions $\phi_{1}\left(x, \lambda_{0}\right)$ and $\chi_{1}\left(x, \lambda_{0}\right)$ are linearly dependent. That is,

$$
\chi_{1}\left(x, \lambda_{0}\right)=k_{1} \phi_{1}\left(x, \lambda_{0}\right), \quad x \in[-1,0)
$$

for some $k_{1} \neq 0$. Then

$$
\begin{aligned}
\alpha_{1} \chi & \left(-1, \lambda_{0}\right)+\alpha_{2} \chi^{\prime}\left(-1, \lambda_{0}\right) \\
& =\alpha_{1} \chi_{1}\left(-1, \lambda_{0}\right)+\alpha_{2} \chi_{1}^{\prime}\left(-1, \lambda_{0}\right) \\
& =k_{1}\left(\alpha_{1} \phi_{1}\left(-1, \lambda_{0}\right)+\alpha_{2} \phi_{1}^{\prime}\left(-1, \lambda_{0}\right)\right)=0 .
\end{aligned}
$$

So, $\chi\left(x, \lambda_{0}\right)$ satisfies condition (2). And the solution $\chi\left(x, \lambda_{0}\right)$ satisfies condition (3) and both transmission condition (4) from its construction. Thus $\chi\left(x, \lambda_{0}\right)$ would be an eigenfunction of the S-L problem (1)-(4) for the eigenvalue $\lambda_{0}$.

Lemma 6. The eigenvalues of the $S$-L problem (1)-(4) are simple.

Proof. Let $\lambda=u+i v$. We differentiate the equation $l \chi(x, \lambda)=$ $\lambda \chi(x, \lambda)$ with respect to $\lambda$ and have $l \chi_{\lambda}(x, \lambda)=\lambda \chi_{\lambda}(x, \lambda)+$ $\chi(x, \lambda)$. Then

$$
\begin{aligned}
\left\langle l \chi_{\lambda}, \phi\right\rangle-\left\langle\chi_{\lambda}, l \phi\right\rangle & =\left\langle\lambda \chi_{\lambda}+\chi, \phi\right\rangle-\left\langle\chi_{\lambda}, \lambda \phi\right\rangle \\
& =\langle\chi, \phi\rangle+2 i v\left\langle\chi_{\lambda}, \phi\right\rangle .
\end{aligned}
$$

By integration by parts and some calculations

$$
\begin{aligned}
& \left\langle l \chi_{\lambda}, \phi\right\rangle-\left\langle\chi_{\lambda}, l \phi\right\rangle \\
& =\rho \int_{-1}^{0}\left(-\chi_{\lambda}^{\prime \prime}(x, \lambda)+q(x) \chi_{\lambda}(x, \lambda)\right) \overline{\phi(x, \lambda)} d x \\
& \quad+\int_{0}^{1}\left(-\chi_{\lambda}^{\prime \prime}(x, \lambda)+q(x) \chi_{\lambda}(x, \lambda)\right) \overline{\phi(x, \lambda)} d x
\end{aligned}
$$




$$
\begin{gathered}
-\rho \int_{-1}^{0} \chi_{\lambda}(x, \lambda) \overline{\left(-\phi^{\prime \prime}(x, \lambda)+q(x) \phi(x, \lambda)\right)} d x \\
-\int_{0}^{1} \chi_{\lambda}(x, \lambda) \overline{\left(-\phi^{\prime \prime}(x, \lambda)+q(x) \phi(x, \lambda)\right)} d x \\
=\rho\left[\left.\left(\chi_{1 \lambda}(x, \lambda) \overline{\phi_{1}^{\prime}(x, \lambda)}-\chi_{1 \lambda}^{\prime}(x, \lambda) \overline{\phi_{1}(x, \lambda)}\right)\right|_{-1} ^{0-}\right. \\
\left.\quad+\left.\left(\chi_{2 \lambda}(x, \lambda) \overline{\phi_{2}^{\prime}(x, \lambda)}-\chi_{2 \lambda}^{\prime}(x, \lambda) \overline{\phi_{2}(x, \lambda)}\right)\right|_{0+} ^{1}\right] \\
=\rho\left(\alpha_{2} \chi_{\lambda}^{\prime}(-1, \lambda)+\alpha_{1} \chi_{\lambda}(-1, \lambda)\right)
\end{gathered}
$$

from (13), (14), (16), and (17) where $\chi_{j \lambda}(x, \lambda), \chi_{j \lambda}^{\prime}(x, \lambda)$ are the derivatives of $\chi_{j}(x, \lambda), \chi_{j}^{\prime}(x, \lambda)$ with respect to $\lambda(j=1,2)$. Since $\omega(\lambda)$ is independent of the variable $x$ and the value of $\phi_{1}(-1, \lambda)$ and $\phi_{1}^{\prime}(-1, \lambda)$ given in (13),

$$
\begin{aligned}
\left.\omega^{\prime}(\lambda)\right|_{x=-1}= & \left.\frac{d \omega_{1}(\lambda)}{d \lambda}\right|_{x=-1} \\
= & \frac{d}{d \lambda}\left(\phi_{1}(-1, \lambda) \chi_{1}^{\prime}(-1, \lambda)\right. \\
& \left.\quad-\phi_{1}(-1, \lambda) \chi_{1}^{\prime}(-1, \lambda)\right) \\
= & \alpha_{2} \chi_{1 \lambda}^{\prime}(-1, \lambda)+\alpha_{1} \chi_{1 \lambda}(-1, \lambda) .
\end{aligned}
$$

So, by (24), (25), and (26),

$$
\omega^{\prime}(\lambda)=\frac{1}{\rho}\left(\langle\chi, \phi\rangle+2 i v\left\langle\chi_{\lambda}, \phi\right\rangle\right) .
$$

Let $\lambda_{0}$ be an eigenvalue of the S-L problem (1)-(4). Then $\lambda_{0}$ is real. Since $\omega\left(\lambda_{0}\right)=0$ for the eigenvalue of the S-L problem (1)-(4) from Theorem $5, \chi_{j}\left(x, \lambda_{0}\right)=c_{j} \phi_{j}\left(x, \lambda_{0}\right)$ for some $c_{j} \neq 0(j=1,2)$. And from $(4)$,

$$
\begin{aligned}
\chi_{2}\left(0+, \lambda_{0}\right) & =c_{1}\left(\gamma_{1} \phi_{1}\left(0-, \lambda_{0}\right)+\gamma_{2} \phi_{1}^{\prime}\left(0-, \lambda_{0}\right)\right) \\
& =c_{1} \phi_{2}\left(0+, \lambda_{0}\right), \\
\chi_{2}^{\prime}\left(0+, \lambda_{0}\right) & =c_{1}\left(\gamma_{3} \phi_{1}\left(0-, \lambda_{0}\right)+\gamma_{4} \phi_{1}^{\prime}\left(0-, \lambda_{0}\right)\right) \\
& =c_{1} \phi_{2}^{\prime}\left(0+, \lambda_{0}\right),
\end{aligned}
$$

we get $c_{1}=c_{2} \neq 0$. Thus, (27) becomes

$$
\omega^{\prime}\left(\lambda_{0}\right)=\frac{1}{\rho}\langle\chi, \phi\rangle=\frac{c_{1}}{\rho}\langle\phi, \phi\rangle \neq 0 .
$$

Hence $\lambda_{0}$ is simple.

\section{Asymptotic Formulas for Eigenvalues and Eigenfunctions}

In this section, the asymptotic formulas for eigenvalues and eigenfunctions of the S-L problem (1)-(4) are obtained by using the asymptotic expressions of the solutions. At first, we calculate the asymptotic expressions of the solutions.
Lemma 7. Let $\lambda=s^{2}, s=\sigma+$ it. If $\alpha_{2} \neq 0$, then one has the following asymptotic expressions:

$$
\begin{array}{r}
\frac{d^{k}}{d x^{k}} \phi_{1}(x, \lambda)=\alpha_{2} \frac{d^{k}}{d x^{k}} \cos s(x+1)+O\left(|s|^{k-1} e^{|t|(x+1)}\right), \\
k=0,1, \\
\text { as }|\lambda| \rightarrow \infty . \text { If } \alpha_{2}=0, \text { then } \\
\frac{d^{k}}{d x^{k}} \phi_{1}(x, \lambda)=-\frac{\alpha_{1}}{s} \frac{d^{k}}{d x^{k}} \sin s(x+1)+O\left(|s|^{k-2} e^{|t|(x+1)}\right), \\
k=0,1,
\end{array}
$$

as $|\lambda| \rightarrow \infty$.

Proof. The asymptotic formulas for $\phi_{1}(x, \lambda)$ follow from the similar formulas of Lemma 1.7 in [20].

The asymptotic formulas for $\phi_{2}(x, \lambda)$ are as follows.

Lemma 8. Let $\lambda=s^{2}, s=\sigma+$ it; then $\phi_{2}(x, \lambda)$ has the following asymptotic formulas as $|\lambda| \rightarrow \infty$ :

(1) if $\alpha_{2} \neq 0, \gamma_{2} \neq 0$, then

$\frac{d^{k}}{d x^{k}} \phi_{2}(x, \lambda)=-\alpha_{2} \gamma_{2} s \sin s \frac{d^{k}}{d x^{k}} \cos s x+O\left(e^{|t| x}\right)$,

(2) if $\alpha_{2} \neq 0, \gamma_{2}=0$, then

$$
\begin{aligned}
& \frac{d^{k}}{d x^{k}} \phi_{2}(x, \lambda) \\
& =\alpha_{2}\left(\gamma_{1} \cos s \frac{d^{k}}{d x^{k}} \cos s x-\gamma_{4} \sin s \frac{d^{k}}{d x^{k}} \sin s x\right) \\
& \quad+O\left(|s|^{-1} e^{|t| x}\right),
\end{aligned}
$$

(3) if $\alpha_{2}=0, \gamma_{2} \neq 0$, then

$$
\frac{d^{k}}{d x^{k}} \phi_{2}(x, \lambda)=-\alpha_{1} \gamma_{2} \cos s \frac{d^{k}}{d x^{k}} \cos s x+O\left(|s|^{-1} e^{|t| x}\right),
$$

(4) if $\alpha_{2}=0, \gamma_{2}=0$, then

$$
\begin{aligned}
& \frac{d^{k}}{d x^{k}} \phi_{2}(x, \lambda) \\
& =-\frac{\alpha_{1}}{s}\left(\gamma_{1} \sin s \frac{d^{k}}{d x^{k}} \cos s x+\gamma_{4} \cos s \frac{d^{k}}{d x^{k}} \sin s x\right) \\
& +O\left(|s|^{-2} e^{|t| x}\right)
\end{aligned}
$$

for $k=0,1$. 
Proof. Let $\lambda=s^{2}, s=\sigma+i t . \phi_{2}(x, \lambda)$ satisfies (14). By the method of variation of constants, we have $\phi_{2}(x, \lambda)$ satisfying the following integral equation:

$$
\begin{aligned}
\phi_{2}(x, \lambda)= & \left(\gamma_{1} \phi_{1}(0-, \lambda)+\gamma_{2} \phi_{1}^{\prime}(0-, \lambda)\right) \cos s x \\
& +\frac{1}{s}\left(\gamma_{3} \phi_{1}(0-, \lambda)+\gamma_{4} \phi_{1}^{\prime}(0-, \lambda)\right) \sin s x \\
& +\frac{1}{s} \int_{0}^{x} \sin [s(x-z)] q(z) \phi_{2}(z, \lambda) d z .
\end{aligned}
$$

Let $\alpha_{2} \neq 0$. Substituting (30), for $k=0$, in (36), we have

$$
\begin{aligned}
\phi_{2}(x, \lambda)= & -\alpha_{2} \gamma_{2} s \sin s \cos s x \\
& +\alpha_{2}\left(\gamma_{1} \cos s \cos s x-\gamma_{4} \sin s \sin s x\right) \\
& +\frac{1}{s} \alpha_{2} \gamma_{3} \cos s \sin s x \\
& +\frac{1}{s} \int_{0}^{x} \sin [s(x-z)] q(z) \phi_{2}(z, \lambda) d z \\
& +O\left(\frac{1}{s} e^{|t|(x+1)}\right) .
\end{aligned}
$$

We consider the case $\gamma_{2} \neq 0$. Let $\phi_{2}(x, \lambda)=|s| e^{|t| x} F(x, \lambda)$. Multiplying (37) by $|s|^{-1} e^{-|t| x}$, we have the following:

$$
\begin{aligned}
F(x, \lambda)= & -\alpha_{2} \gamma_{2} e^{-|t| x} \sin s \cos s x \\
& +\frac{1}{|s|} \alpha_{2} e^{-|t| x}\left(\gamma_{1} \cos s \cos s x-\gamma_{4} \sin s \sin s x\right) \\
& +\frac{1}{|s|^{2}} \alpha_{2} \gamma_{3} e^{-|t| x} \cos s \sin s x \\
& +\frac{1}{|s|} \int_{0}^{x} \sin [s(x-z)] e^{-|t|(x-z)} q(z) F(z, \lambda) d z \\
& +O\left(\frac{1}{|s|^{2}} e^{|t|}\right) .
\end{aligned}
$$

Let $\mu(\lambda)=\max _{-1 \leq x \leq 1}|F(x, \lambda)|$. Then

$$
\mu(\lambda) \leq\left|\alpha_{2} \gamma_{2}\right|+\frac{M_{0}}{|s|}
$$

for some $M_{0}>0$. Consequently, $\mu(\lambda)=O(1)$ as $|\lambda| \rightarrow \infty$. So, $\phi_{2}(x, \lambda)=|s| e^{|t| x} F(x, \lambda)=O\left(|s| e^{|t| x}\right)$ as $|\lambda| \rightarrow \infty$. Substituting the asymptotic equality in (37) gives (32) for $k=0$. And the case for $k=1$ is obtained by differentiating (37) and by some similar calculations.

Similarly, we can prove (33), (34), and (35).

When $\lambda$ is big enough, the asymptotic formulas for $\phi_{2}(x, \lambda)$ are obtained in Lemma 8. Substituting the asymptotic formulas of $\phi_{2}(x, \lambda)$ and the value of $\chi_{2}(x, \lambda)$ at $x=$ 1 , which is given in (16), into $\omega(\lambda)=(1 / \rho) \omega_{2}(\lambda)$, we can establish the following lemma.
Lemma 9. Let $\lambda=s^{2}, s=\sigma+i$; then $\omega(\lambda)$ has the following asymptotic formulas for large enough $\lambda$.

(1) If $\alpha_{2} \neq 0, \gamma_{2} \neq 0, \beta_{2} \neq 0$, then

$$
\omega(\lambda)=-\frac{1}{\rho} \alpha_{2} \beta_{2} \gamma_{2} s^{2} \sin ^{2} s+O\left(|s| e^{|t|}\right) .
$$

(2) If $\alpha_{2} \neq 0, \gamma_{2} \neq 0, \beta_{2}=0$, then

$$
\omega(\lambda)=\frac{1}{\rho} \alpha_{2} \beta_{1} \gamma_{2} s \sin s \cos s+O\left(e^{|t|}\right) .
$$

(3) If $\alpha_{2} \neq 0, \gamma_{2}=0, \beta_{2} \neq 0$, then

$$
\omega(\lambda)=\frac{1}{\rho} \alpha_{2} \beta_{2}\left(\gamma_{1}+\gamma_{4}\right) s \sin s \cos s+O\left(e^{|t|}\right) .
$$

(4) If $\alpha_{2} \neq 0, \gamma_{2}=0, \beta_{2}=0$, then

$$
\omega(\lambda)=\frac{1}{\rho} \alpha_{2} \beta_{1}\left(\gamma_{4} \sin ^{2} s-\gamma_{1} \cos ^{2} s\right)+O\left(|s|^{-1} e^{|t|}\right) .
$$

(5) If $\alpha_{2}=0, \gamma_{2} \neq 0, \beta_{2} \neq 0$, then

$$
\omega(\lambda)=-\frac{1}{\rho} \alpha_{1} \beta_{2} \gamma_{2} s \sin s \cos s+O\left(e^{|t|}\right) .
$$

(6) If $\alpha_{2}=0, \gamma_{2} \neq 0, \beta_{2}=0$, then

$$
\omega(\lambda)=\frac{1}{\rho} \alpha_{1} \beta_{1} \gamma_{2} \cos ^{2} s+O\left(|s|^{-1} e^{|t|}\right) .
$$

(7) If $\alpha_{2}=0, \gamma_{2}=0, \beta_{2} \neq 0$, then

$\omega(\lambda)=\frac{1}{\rho} \alpha_{1} \beta_{2}\left(\gamma_{1} \sin ^{2} s-\gamma_{4} \cos ^{2} s\right)+O\left(|s|^{-1} e^{|t|}\right)$.

(8) If $\alpha_{2}=0, \gamma_{2}=0, \beta_{2}=0$, then

$$
\omega(\lambda)=\frac{1}{\rho s} \alpha_{1} \beta_{1}\left(\gamma_{1}+\gamma_{4}\right) \sin s \cos s+O\left(|s|^{-2} e^{|t|}\right) .
$$

Theorem 10. The eigenvalues of the S-L problem (1)-(4) are bounded below and countably infinite; the unique cluster point is infinity.

Proof. From Theorem 5, the eigenvalues of the S-L problem (1)-(4) are zeros of the function $\omega(\lambda)$ which is an entire function of $\lambda$. And the asymptotic formulas of the function $\omega(\lambda)$ are obtained in Lemma 9. Let

$$
\omega(\lambda)=\omega^{*}(\lambda)+\delta(\lambda),
$$

where $\omega^{*}(\lambda)=-(1 / \rho) \alpha_{2} \beta_{2} \gamma_{2} s^{2} \sin ^{2} s, \delta(\lambda)=O\left(|s| e^{|t|}\right)$ for the case $\alpha_{2} \neq 0, \gamma_{2} \neq 0, \beta_{2} \neq 0$ from Lemma 9 .

Let $\lambda=s^{2}, s=\sigma+$ it and let

$$
\begin{aligned}
& \Gamma_{n}^{\prime}=\left\{\lambda=s^{2}=(\sigma+i t)^{2}|| \sigma \mid=\left(n+\frac{1}{2}\right) \pi, 0 \leq t \leq n\right\}, \\
& \Gamma_{n}^{\prime \prime}=\left\{\lambda=s^{2}=(\sigma+i t)^{2}|| \sigma \mid \leq\left(n+\frac{1}{2}\right) \pi, t=n\right\},
\end{aligned}
$$


and let $\Gamma_{n}=\Gamma_{n}^{\prime} \cup \Gamma_{n}^{\prime \prime}$. Then $\Gamma_{n}$ is a closed curve on the plane of $\lambda$. Next, we prove that $\left|\omega^{*}(\lambda)\right|>|\delta(\lambda)|$ on $\Gamma_{n}$. When $|\lambda|$ is big enough, on $\Gamma_{n}^{\prime}$

$$
\begin{aligned}
|\sin s| & =\frac{1}{2}\left|e^{i s}-e^{-i s}\right|=\frac{1}{2}\left|e^{i(n+(1 / 2)) \pi} e^{-t}-e^{-i(n+(1 / 2)) \pi} e^{t}\right| \\
& =\frac{1}{2}\left|i e^{-t}+i e^{t}\right|=\frac{1}{2} e^{t}\left|1+e^{-2 t}\right|>\frac{e^{|t|}}{4} .
\end{aligned}
$$

Similarly, when $|\lambda|$ is big enough, on $\Gamma_{n}^{\prime \prime}$

$$
\begin{aligned}
|\sin s| & =\frac{1}{2}\left|e^{i \sigma \pi} e^{-n}-e^{-i \sigma \pi} e^{n}\right| \\
& =\frac{1}{2} e^{n}\left|1-e^{2 i \sigma \pi} e^{-2 n}\right|>\frac{e^{n}}{4} .
\end{aligned}
$$

We obtain

$$
\begin{aligned}
\left|\omega^{*}(\lambda)\right|_{\Gamma_{n}} & =\left|-\frac{1}{\rho} \alpha_{2} \beta_{2} \gamma_{2} s^{2} \sin ^{2} s\right|_{\Gamma_{n}} \\
& >\frac{n}{4 \rho} e^{|t|}\left|\alpha_{2} \beta_{2} \gamma_{2} s \sin s\right| .
\end{aligned}
$$

So,

$$
\left|\omega^{*}(\lambda)\right|_{\Gamma_{n}}>|\delta(\lambda)|_{\Gamma_{n}}
$$

By Rouche's Theorem in [21], $\omega(\lambda)$ and $\omega^{*}(\lambda)$ have the same zeros interior of $\Gamma_{n}=\Gamma_{n}^{\prime} \cup \Gamma_{n}^{\prime \prime}$ as follows:

$$
0^{2}, \pi^{2},(2 \pi)^{2}, \ldots,(n \pi)^{2} \text {. }
$$

Letting $s=i t$, we can prove that $\left|\omega^{*}(\lambda)\right|_{\Gamma_{n}}>|\delta(\lambda)|_{\Gamma_{n}}$. Hence, $\omega(\lambda)=\omega\left(-t^{2}\right) \neq 0$ as $|t| \rightarrow \infty$. Thus, $\omega(\lambda)$ only has finite negative zeros. The proof of other cases in Lemma 9 is similar to the proof of the case $\alpha_{2} \neq 0, \gamma_{2} \neq 0, \beta_{2} \neq 0$.

Theorem 11. Let $\lambda_{1}, \lambda_{2}, \lambda_{3}, \ldots$, be the collection of all eigenvalues of the $S$-L problem (1)-(4) and let $\varphi_{1}(x), \varphi_{2}(x), \ldots$ be the corresponding normalized eigenfunctions. Then

$$
-\infty<\lambda_{1} \leq \lambda_{2} \leq \cdots \lambda_{n} \cdots \longrightarrow \infty .
$$

And $\left\{\varphi_{n} ; n \in \mathbb{N}\right\}$ is complete in $H$ and

$$
\left\langle\varphi_{n}, \varphi_{m}\right\rangle= \begin{cases}1, & n=m, \\ 0, & n \neq m .\end{cases}
$$

Theorem 12. The following asymptotic formulas hold for eigenvalues and eigenfunctions of the S-L problem (1)-(4) for large enough $n \in \mathbb{N}$.

(1) If $\alpha_{2} \neq 0, \gamma_{2} \neq 0, \beta_{2} \neq 0$, then

$$
\begin{gathered}
\sqrt{\lambda_{n}}=(n-1) \pi+O\left(\frac{1}{n}\right), \\
\phi\left(x, \lambda_{n}\right)= \begin{cases}\alpha_{2} \cos (n-1) \pi(x+1)+O\left(\frac{1}{n}\right), & x \in[-1,0), \\
O(1), & x \in(0,1] .\end{cases}
\end{gathered}
$$

(2) If $\alpha_{2} \neq 0, \gamma_{2} \neq 0, \beta_{2}=0$, then

$$
\begin{gathered}
\sqrt{\lambda_{n}}=\frac{(n-1)}{2} \pi+O\left(\frac{1}{n}\right), \\
\qquad\left(x, \lambda_{n}\right)=\left\{\begin{array}{cc}
\alpha_{2} \cos \frac{(n-1)}{2} \pi(x+1) \\
+O\left(\frac{1}{n}\right), & x \in[-1,0), \\
\alpha_{2} \gamma_{2} \frac{(n-1)}{2} \pi \cos \frac{(n-1)}{2} \pi x & \\
+O(1)(k \text { is even }), & \\
O(1)(k \text { is odd }), & x \in(0,1] .
\end{array}\right.
\end{gathered}
$$

(3) If $\alpha_{2} \neq 0, \gamma_{2}=0, \beta_{2} \neq 0$, then

$$
\begin{gathered}
\sqrt{\lambda_{n}}=\frac{(n-1)}{2} \pi+O\left(\frac{1}{n}\right), \\
\phi\left(x, \lambda_{n}\right)= \begin{cases}\alpha_{2} \cos \frac{(n-1)}{2} \pi(x+1)+O\left(\frac{1}{n}\right), & x \in[-1,0), \\
O\left(\frac{1}{n}\right), & x \in(0,1] .\end{cases}
\end{gathered}
$$

(4) If $\alpha_{2} \neq 0, \gamma_{2}=0, \beta_{2}=0$, then

$$
\sqrt{\lambda_{n}}=(n-1) \pi \pm \arctan \sqrt{\frac{\gamma_{1}}{\gamma_{4}}}+O\left(\frac{1}{n}\right)
$$

$\phi\left(x, \lambda_{n}\right)$

$$
\left\{\begin{array}{c}
\alpha_{2} \cos \left[(n-1) \pi \pm \arctan \sqrt{\frac{\gamma_{1}}{\gamma_{4}}}\right] \\
\times(x+1)+O\left(\frac{1}{n}\right), \quad x \in[-1,0), \\
\alpha_{2}\left(\gamma_{1} \cos \left[(n-1) \pi \pm \arctan \sqrt{\frac{\gamma_{1}}{\gamma_{4}}}\right]\right. \\
\times \cos \left[(n-1) \pi \pm \arctan \sqrt{\left.\frac{\gamma_{1}}{\gamma_{4}}\right] x}\right. \\
-\gamma_{4} \sin \left[(n-1) \pi \pm \arctan \sqrt{\frac{\gamma_{1}}{\gamma_{4}}}\right] \\
\left.\times \sin \left[(n-1) \pi \pm \arctan \sqrt{\frac{\gamma_{1}}{\gamma_{4}}}\right] x\right), \\
x \in(0,1] .
\end{array}\right.
$$


(5) If $\alpha_{2}=0, \gamma_{2} \neq 0, \beta_{2} \neq 0$, then

$$
\phi\left(x, \lambda_{n}\right)=\left\{\begin{array}{cc}
-\frac{2 \alpha_{1}}{(n-1) \pi} \sin \frac{(n-1)}{2} \pi(x+1) & \\
+O\left(\frac{1}{\lambda^{2}}\right), & x \in[-1,0), \\
\alpha_{1} \gamma_{2} \cos \frac{(n-1)}{2} \pi x & \\
+O\left(\frac{1}{n}\right)(k \text { is odd }) & \\
O\left(\frac{1}{n}\right)(k \text { is even }), & x \in(0,1] .
\end{array}\right.
$$

(6) If $\alpha_{2}=0, \gamma_{2} \neq 0, \beta_{2}=0$, then

$$
\begin{gathered}
\sqrt{\lambda_{n}}=\left(n-\frac{1}{2}\right) \pi+O\left(\frac{1}{n}\right), \\
\phi\left(x, \lambda_{n}\right)= \begin{cases}-\frac{\alpha_{1}}{(n-(1 / 2)) \pi} \sin \left(n-\frac{1}{2}\right) \\
\times \pi(x+1)+O\left(\frac{1}{n^{2}}\right), & x \in[-1,0), \\
O\left(\frac{1}{n}\right), & x \in(0,1] .\end{cases}
\end{gathered}
$$

(7) If $\alpha_{2}=0, \gamma_{2}=0, \beta_{2} \neq 0$, then

$$
\begin{aligned}
& \sqrt{\lambda_{n}}=(n-1) \pi \pm \arctan \sqrt{\frac{\gamma_{4}}{\gamma_{1}}}+O\left(\frac{1}{n}\right), \\
& \phi\left(x, \lambda_{n}\right) \\
& =\left\{\begin{array}{l}
-\frac{\alpha_{1}}{(n-1) \pi \pm \arctan \sqrt{\gamma_{4} / \gamma_{1}}} \\
\times \sin \left[(n-1) \pi \pm \arctan \sqrt{\frac{\gamma_{4}}{\gamma_{1}}}\right] \\
\times(x+1)+O\left(\frac{1}{n^{2}}\right), \quad x \in[-1,0), \\
-\frac{\alpha_{1}}{(n-1) \pi \pm \arctan \sqrt{\gamma_{4} / \gamma_{1}}} \\
\times\left(\gamma_{1} \sin \left[(n-1) \pi \pm \arctan \sqrt{\frac{\gamma_{4}}{\gamma_{1}}}\right]\right. \\
\times \cos \left[(n-1) \pi \pm \arctan \sqrt{\frac{\gamma_{4}}{\gamma_{1}}}\right] x \\
+\gamma_{4} \cos \left[(n-1) \pi \pm \arctan \sqrt{\frac{\gamma_{4}}{\gamma_{1}}}\right] \\
\left.\times \sin \left[(n-1) \pi \pm \arctan \sqrt{\frac{\gamma_{4}}{\gamma_{1}}}\right] x\right) \\
+O\left(\frac{1}{n^{2}}\right), \quad x \in(0,1] .
\end{array}\right.
\end{aligned}
$$

(8) If $\alpha_{2}=0, \gamma_{2}=0, \beta_{2}=0$, then

$$
\begin{aligned}
& \sqrt{\lambda_{n}}=\frac{(n-1)}{2} \pi+O\left(\frac{1}{n}\right),
\end{aligned}
$$

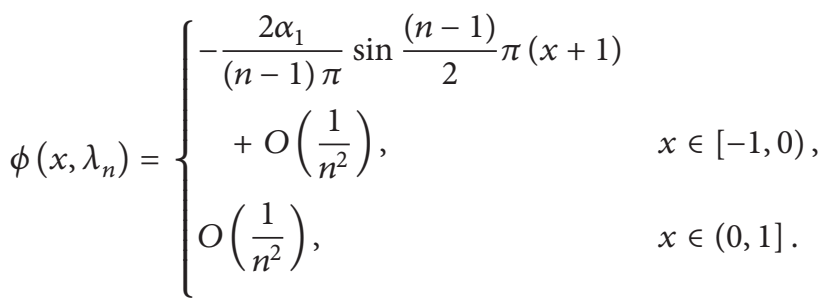

Proof. By Theorem 5, we know that the eigenvalues of the S-L problem (1)-(4) coincide with the zeros of the function $\omega(\lambda)$. From Lemma 9 and Theorem 10, the entire function $\omega(\lambda)$ has a sequence of zeros:

$$
\sqrt{\lambda_{n}}=(n-1) \pi+O\left(\frac{1}{n}\right),
$$

for the case $\alpha_{2} \neq 0, \gamma_{2} \neq 0, \beta_{2} \neq 0$ when $n$ is big enough. By using the asymptotic formulas of eigenvalues $\lambda_{n}$, the corresponding eigenfunctions $\phi\left(x, \lambda_{n}\right)$ have the following asymptotic formulas:

$$
\phi\left(x, \lambda_{n}\right)= \begin{cases}\alpha_{2} \cos (n-1) \pi(x+1)+O\left(\frac{1}{n}\right), & x \in[-1,0), \\ O(1), & x \in(0,1]\end{cases}
$$

for the case $\alpha_{2} \neq 0, \gamma_{2} \neq 0, \beta_{2} \neq 0$. Similarly, we can obtain the other cases.

By the asymptotic formulas for the eigenvalues and eigenfunctions of the S-L problem (1)-(4) in the above theorem, we know that the series $\sum_{n=1}^{\infty}\left(\varphi_{n}(x) \overline{\varphi_{n}(\xi)} / \lambda_{n}\right)$ converges absolutely and uniformly where $\varphi_{n}(x)$ is the normalized eigenfunction.

Green's function of the classical S-L problem is studied in [22]. Here, we obtain Green's function of the S-L problem (1)(4) as follows:

$$
\begin{aligned}
& G(x, \xi, \lambda) \\
& \quad= \begin{cases}-\frac{1}{\rho} \frac{\phi_{1}(\xi, \lambda) \chi_{1}(x, \lambda)}{\omega_{1}}, & -1 \leq \xi \leq x<0, \\
-\frac{1}{\rho} \frac{\phi_{1}(x, \lambda) \chi_{1}(\xi, \lambda)}{\omega_{1}}, & -1 \leq x \leq \xi<0, \\
-\frac{1}{\rho} \frac{\phi_{1}(x, \lambda) \chi_{2}(\xi, \lambda)}{\omega_{2}}, & -1 \leq x<0,0<\xi \leq 1, \\
-\frac{\phi_{1}(\xi, \lambda) \chi_{2}(x, \lambda)}{\omega_{1}}, & -1 \leq \xi<0,0<x \leq 1, \\
-\frac{\phi_{2}(\xi, \lambda) \chi_{2}(x, \lambda)}{\omega_{2}}, & 0<\xi \leq x \leq 1, \\
-\frac{\phi_{2}(x, \lambda) \chi_{2}(\xi, \lambda)}{\omega_{2}}, & 0<x \leq \xi \leq 1 .\end{cases}
\end{aligned}
$$


Let us consider the nonhomogeneous differential equation

$$
l y-\lambda y=f(x)
$$

together with boundary and transmission conditions (2)-(4) where $f \in H$. Green's functions provide a powerful method for solving the linear nonhomogeneous equations.

Theorem 13. Let $f \in H$. Then the function

$$
y(x, \lambda)=\rho \int_{-1}^{0} G(x, \xi, \lambda) f(\xi) d \xi+\int_{0}^{1} G(x, \xi, \lambda) f(\xi) d \xi
$$

satisfies (69) and both boundary and transmission conditions (2)-(4), where $G(x, \xi, \lambda)$ is the Green function defined in (68).

Proof. Putting (68) in (70), we have

$$
y(x, \lambda)=\left\{\begin{array}{l}
-\frac{1}{\omega_{1}} \chi_{1}(x, \lambda) \int_{-1}^{x} \phi_{1}(\xi, \lambda) f(\xi) d \xi \\
-\frac{1}{\omega_{1}} \phi_{1}(x, \lambda) \int_{x}^{0} \chi_{1}(\xi, \lambda) f(\xi) d \xi \\
-\frac{1}{\omega_{2}} \phi_{1}(x, \lambda) \int_{0}^{1} \chi_{2}(\xi, \lambda) f(\xi) d \xi, \quad x \in[-1,0), \\
-\frac{1}{\omega_{1}} \chi_{2}(x, \lambda) \int_{-1}^{0} \phi_{1}(\xi, \lambda) f(\xi) d \xi \\
-\frac{1}{\omega_{2}} \chi_{2}(x, \lambda) \int_{0}^{x} \phi_{2}(\xi, \lambda) f(\xi) d \xi \\
-\frac{1}{\omega_{2}} \phi_{2}(x, \lambda) \int_{x}^{1} \chi_{2}(\xi, \lambda) f(\xi) d \xi, \quad x \in(0,1] .
\end{array}\right.
$$

By differentiating twice $y(x, \lambda)$ and by the definitions of solutions $\phi_{j}(x, \lambda)$ and $\chi_{j}(x, \lambda)(j=1,2)$, we obtain $y^{\prime \prime}(x, \lambda)=$ $(q(x)-\lambda) y(x, \lambda)-f(x)$. So, the function $y(x, \lambda)$ defined in (70) is the solution of (69). And by calculations, we may prove that (71) satisfies both boundary and transmission conditions (2)-(4).

\section{Eigenfunction Expansion for Green's Function and the Modified Parseval Equality}

In this section, we derive the eigenfunction expansion for Green's function of the S-L problem (1)-(4) and establish the modified Parseval equality in the associated Hilbert space $H$. Without loss of generality, we assume that $\lambda=0$ is not an eigenvalue. Let $G(x, \xi)=G(x, \xi, 0)$.

Theorem 14. Let $\lambda_{n}$ be the eigenvalue of the S-L problem (1)(4) and let $\varphi_{n}(x)$ be the corresponding normalized eigenfunction. Then,

$$
G(x, \xi)=-\sum_{n=1}^{\infty} \frac{\varphi_{n}(x) \overline{\varphi_{n}(\xi)}}{\lambda_{n}}
$$

In order to prove Theorem 14, we need the following lemma.

Lemma 15. The S-L problem (1)-(4) is equivalent to

$$
y(x, \lambda)-\lambda\left(\rho \int_{-1}^{0} G(x, \xi) y(\xi) d \xi+\int_{0}^{1} G(x, \xi) y(\xi) d \xi\right)=0
$$

Proof. By Theorem 13, we know that

$$
y(x, \lambda)=\rho \int_{-1}^{0} G(x, \xi) f(\xi) d \xi+\int_{0}^{1} G(x, \xi) f(\xi) d \xi
$$

satisfies $-y^{\prime \prime}(x)+q(x) y(x)=f(x)$ and both boundary and transmission conditions (2)-(4). Equation (69) can be written in the form $-y^{\prime \prime}(x)+q(x) y(x)=\widetilde{f}(x)$ where $\widetilde{f}(x)=f(x)+$ $\lambda y$. Similar to (74), the new form of (69) has a solution

$$
y(x, \lambda)=\rho \int_{-1}^{0} G(x, \xi) \widetilde{f}(\xi) d \xi+\int_{0}^{1} G(x, \xi) \widetilde{f}(\xi) d \xi,
$$

which satisfies both boundary and transmission conditions (2)-(4). If $f(x) \equiv 0$, then the corresponding homogeneous case is the S-L problem (1)-(4). Consequently, the S-L problem (1)-(4) is equivalent to

$$
y(x, \lambda)-\lambda\left(\rho \int_{-1}^{0} G(x, \xi) y(\xi) d \xi+\int_{0}^{1} G(x, \xi) y(\xi) d \xi\right)=0
$$

Proof of Theorem 14. Let $\lambda_{n}$ be the eigenvalue of the S-L problem (1)-(4) and let $\varphi_{n}(x)$ be the corresponding normalized eigenfunction as in Theorem 11. Let $P(x, \xi)=$ $G(x, \xi)+\sum_{n=1}^{\infty}\left(\varphi_{n}(x) \overline{\varphi_{n}(\xi)} / \lambda_{n}\right)$. Then $P(x, \xi)$ is continuous and symmetric. We assume that $P(x, \xi) \neq 0$. Then, by the Fredholm integral equation, there are a number $\tilde{\lambda}$ and a function $\widetilde{y}(x) \neq 0$ in $H$ such that

$$
\tilde{y}(x)=\tilde{\lambda}\left(\rho \int_{-1}^{0} P(x, \xi) \tilde{y}(\xi) d \xi+\int_{0}^{1} P(x, \xi) \tilde{y}(\xi) d \xi\right) .
$$

By Lemma 15,

$$
\begin{aligned}
\varphi_{n}(x)-\lambda_{n}\left(\rho \int_{-1}^{0} G(x, \xi) \varphi_{n}(\xi) d \xi\right. \\
\left.\quad+\int_{0}^{1} G(x, \xi) \varphi_{n}(\xi) d \xi\right)=0 .
\end{aligned}
$$

Putting $G(x, \xi)=P(x, \xi)-\sum_{n=1}^{\infty}\left(\varphi_{n}(x) \overline{\varphi_{n}(\xi)} / \lambda_{n}\right)$ in (78), we obtain

$$
\rho \int_{-1}^{0} P(x, \xi) \varphi_{n}(\xi) d \xi+\int_{0}^{1} P(x, \xi) \varphi_{n}(\xi) d \xi=0 .
$$


In the following, we prove that $\left\langle\tilde{y}, \varphi_{n}\right\rangle=0$ and $\tilde{y}$ is an eigenfunction of the S-L problem (1)-(4). By (77) and (79)

$$
\begin{aligned}
\left\langle\tilde{y}, \varphi_{n}\right\rangle= & \rho \int_{-1}^{0} \tilde{y}(x) \overline{\varphi_{n}(x)} d x+\int_{0}^{1} \tilde{y}(x) \overline{\varphi_{n}(x)} d x \\
= & \tilde{\lambda} \rho \int_{-1}^{0}\left(\rho \int_{-1}^{0} P(x, \xi) \tilde{y}(\xi) d \xi\right. \\
& \left.+\int_{0}^{1} P(x, \xi) \tilde{y}(\xi) d \xi\right) \overline{\varphi_{n}(x)} d x \\
+ & \tilde{\lambda} \int_{0}^{1}\left(\rho \int_{-1}^{0} P(x, \xi) \tilde{y}(\xi) d \xi\right. \\
= & \left.+\int_{0}^{1} P(x, \xi) \tilde{y}(\xi) d \xi\right) \overline{\varphi_{n}(x)} d x \\
& +\int_{-1}^{0}\left(\rho \int_{-1}^{0} P(x, \xi) \overline{\varphi_{n}(x)} d x\right. \\
& +\tilde{\lambda} \int_{0}^{1}\left(\rho \int_{-1}^{1} P(x, \xi) \overline{\varphi_{n}(x)} d x\right) \tilde{y}(\xi) d \xi \overline{\varphi_{n}(x)} d x \\
& \left.\quad+\int_{0}^{1} P(x, \xi) \overline{\varphi_{n}(x)} d x\right) \tilde{y}(\xi) d \xi=0 .
\end{aligned}
$$

And we have

$$
\begin{gathered}
\tilde{y}(x)-\tilde{\lambda}\left(\rho \int_{-1}^{0} G(x, \xi) \tilde{y}(\xi) d \xi+\int_{0}^{1} G(x, \xi) \tilde{y}(\xi) d \xi\right) \\
=\tilde{y}(x)-\tilde{\lambda}\left(\rho \int_{-1}^{0}\left(P(x, \xi)-\sum_{n=1}^{\infty} \frac{\varphi_{n}(x) \overline{\varphi_{n}(\xi)}}{\lambda_{n}}\right) \tilde{y}(\xi) d \xi\right. \\
\left.\quad+\int_{0}^{1}\left(P(x, \xi)-\sum_{n=1}^{\infty} \frac{\varphi_{n}(x) \overline{\varphi_{n}(\xi)}}{\lambda_{n}}\right) \tilde{y}(\xi) d \xi\right) \\
=\tilde{y}(x)-\tilde{\lambda}\left(\left(\rho \int_{-1}^{0} P(x, \xi) \tilde{y}(\xi) d \xi+\int_{0}^{1} P(x, \xi) \tilde{y}(\xi) d \xi\right)\right. \\
\left.\quad-\sum_{n=1}^{\infty} \frac{\varphi_{n}(x)}{\lambda_{n}}\left\langle\tilde{y}, \varphi_{n}\right\rangle\right) \\
=\tilde{y}(x)-\tilde{\lambda}\left(\rho \int_{-1}^{0} P(x, \xi) \tilde{y}(\xi) d \xi\right. \\
\left.\quad+\int_{0}^{1} P(x, \xi) \tilde{y}(\xi) d \xi\right)=0
\end{gathered}
$$

by (77). This implies that $\tilde{y}$ is the eigenfunction of the SL problem (1)-(4) by Lemma 15. So, by $\left\langle\tilde{y}, \varphi_{n}\right\rangle=0$ and the completeness of the eigenfunctions in Theorem 11, we know that $\tilde{y}=0$. Consequently, $P(x, \xi)=0$. The proof is completed.
Lemma 16. Let $\widetilde{C}_{0}^{\infty}$ be the set of all functions defined by

$$
\psi(x)= \begin{cases}\psi_{1}(x) & x \in[-1,0), \\ \psi_{2}(x) & x \in(0,1],\end{cases}
$$

where $\psi_{1}(x) \in C_{0}^{\infty}[-1,0), \psi_{2}(x) \in C_{0}^{\infty}(0,1]$. Then $\widetilde{C}_{0}^{\infty}$ is dense in $H$.

Proof. Let $f$ be any function in $L^{2}(I)$ with $f(x)=f_{1}(x), x \in$ $[-1,0)$, and $f(x)=f_{2}(x), x \in(0,1]$. Since $C_{0}^{\infty}[-1,0)$ is dense in $L^{2}[-1,0)$ as in [23], for $f_{1} \in L^{2}[-1,0)$, there exists a function $g_{1} \in C_{0}^{\infty}[-1,0)$ such that

$$
\rho \int_{-1}^{0}\left|f_{1}(x)-g_{1}(x)\right|^{2} d x<\frac{\epsilon}{2} .
$$

Similarly, for $f_{2} \in L^{2}(0,1]$, there exists a function $g_{2} \in$ $C_{0}^{\infty}(0,1]$ such that

$$
\int_{0}^{1}\left|f_{2}(x)-g_{2}(x)\right|^{2} d x<\frac{\epsilon}{2} .
$$

Then, for any $f \in H$ and $\epsilon>0$, there exists $g \in \widetilde{C}_{0}^{\infty}$ with $g(x)=\left\{\begin{array}{ll}g_{1}(x) & x \in[-1,0), \\ g_{2}(x) & x \in(0,1]\end{array}\right.$ such that

$$
\begin{aligned}
\|f-g\|_{H}^{2}= & \rho \int_{-1}^{0}\left|f_{1}(x)-g_{1}(x)\right|^{2} d x \\
& +\int_{0}^{1}\left|f_{2}(x)-g_{2}(x)\right|^{2} d x<\epsilon .
\end{aligned}
$$

Thus, $\widetilde{C}_{0}^{\infty}$ is dense in $H$.

In the following theorem, we prove the modified Parseval equality in the associated Hilbert space $H$.

Theorem 17. Let $f \in H$. Then, the modified Parseval equality holds; that is,

$$
\|f\|_{H}^{2}=\sum_{n=1}^{\infty} c_{n}^{2}(f)
$$

where $\|f\|_{H}^{2}=\langle f, f\rangle$ and

$$
c_{n}(f)=\rho \int_{-1}^{0} f(x) \overline{\varphi_{n}(x)} d x+\int_{0}^{1} f(x) \overline{\varphi_{n}(x)} d x .
$$

Proof. Let $\widetilde{C}_{0}^{\infty}$ be as in Lemma 16. At first, we prove that (86) holds for $f \in \widetilde{C}_{0}^{\infty}$. Denote $g(x)=-f^{\prime \prime}(x)+q(x) f$. Then by Theorems 13 and 14 ,

$$
\begin{aligned}
f(x)= & \rho \int_{-1}^{0} G(x, \xi) g(\xi) d \xi+\int_{0}^{1} G(x, \xi) g(\xi) d \xi \\
= & -\sum_{n=1}^{\infty} \frac{1}{\lambda_{n}} \varphi_{n}(x) \\
& \times\left(\rho \int_{-1}^{0} \overline{\varphi_{n}(\xi)} g(\xi) d \xi+\int_{0}^{1} \overline{\varphi_{n}(\xi)} g(\xi) d \xi\right) .
\end{aligned}
$$


Multiplying by $\overline{\varphi_{m}(x)}$ and integrating the new equation, we have

$$
\begin{aligned}
& \rho \int_{-1}^{0} \overline{\varphi_{m}(x)} f(x) d x+\int_{0}^{1} \overline{\varphi_{m}(x)} f(x) d x \\
& \quad=-\frac{1}{\lambda_{m}}\left(\rho \int_{-1}^{0} \overline{\varphi_{m}(\xi)} g(\xi) d \xi+\int_{0}^{1} \overline{\varphi_{m}(\xi)} g(\xi) d \xi\right) .
\end{aligned}
$$

Then

$$
f(x)=\sum_{n=1}^{\infty} c_{n}(f) \varphi_{n}(x)
$$

where $c_{n}(f)=\left\langle f, \varphi_{n}\right\rangle=\rho \int_{-1}^{0} f(x) \overline{\varphi_{n}(x)} d x+\int_{0}^{1} f(x)$ $\overline{\varphi_{n}(x)} d x$. Thus, for $f \in \widetilde{C}_{0}^{\infty}$,

$$
\|f\|_{H}^{2}=\sum_{n=1}^{\infty} c_{n}^{2}(f) .
$$

Next, we prove that (86) holds for $f \in H$. For any $f \in H$, there exists a sequence $\left\{f_{k}\right\}_{k \in \mathbb{N}} \subset \widetilde{C}_{0}^{\infty}$ converging to $f$ in $H$ since $\widetilde{C}_{0}^{\infty}$ is dense in $H$. Firstly, we prove that $\sum_{n=1}^{\infty} c_{n}^{2}(f)<\infty$ and $\lim _{k \rightarrow \infty} \sum_{n=1}^{\infty} c_{n}^{2}\left(f_{k}\right)=\sum_{n=1}^{\infty} c_{n}^{2}(f)$.

By the Cauchy-Schwartz inequality, $\left|c_{n}\left(f_{k}\right)-c_{n}(f)\right|=$ $\left|\left\langle f_{k}-f, \varphi_{n}\right\rangle\right| \leq\left\|f_{k}-f\right\|_{H}$. Hence, $\lim _{k \rightarrow \infty} c_{n}\left(f_{k}\right)=c_{n}(f)$. Since $\sum_{n=1}^{\infty}\left(c_{n}\left(f_{k}\right)-c_{n}\left(f_{m}\right)\right)^{2}=\sum_{n=1}^{\infty} c_{n}^{2}\left(f_{k}-f_{m}\right)=\left\|f_{k}-f_{m}\right\|_{H}^{2}$ for $f_{k}-f_{m} \in \widetilde{C}_{0}^{\infty}$,

$$
\sum_{n=1}^{N}\left(c_{n}\left(f_{k}\right)-c_{n}\left(f_{m}\right)\right)^{2} \leq\left\|f_{k}-f_{m}\right\|_{H}^{2}
$$

Let $k \rightarrow \infty$, then inequality (92) becomes $\sum_{n=1}^{N}\left(c_{n}(f)-\right.$ $\left.c_{n}\left(f_{m}\right)\right)^{2} \leq\left\|f-f_{m}\right\|_{H}^{2}$. Letting $N \rightarrow \infty$, we have

$$
\sum_{n=1}^{\infty}\left(c_{n}(f)-c_{n}\left(f_{m}\right)\right)^{2} \leq\left\|f-f_{m}\right\|_{H^{*}}^{2}
$$

Then by the Minkowski inequality

$$
\begin{aligned}
\sum_{n=1}^{\infty} c_{n}^{2}(f)= & \sum_{n=1}^{\infty}\left(c_{n}(f)-c_{n}\left(f_{m}\right)+c_{n}\left(f_{m}\right)\right)^{2} \\
\leq & \left(\left(\sum_{n=1}^{\infty}\left(c_{n}(f)-c_{n}\left(f_{m}\right)\right)^{2}\right)^{1 / 2}\right. \\
& \left.+\left(\sum_{n=1}^{\infty} c_{n}^{2}\left(f_{m}\right)\right)^{1 / 2}\right)^{2}<\infty
\end{aligned}
$$

and by Hölder's inequality,

$$
\begin{aligned}
& \left|\sum_{n=1}^{\infty} c_{n}^{2}(f)-\sum_{n=1}^{\infty} c_{n}^{2}\left(f_{k}\right)\right| \\
& \quad=\left|\sum_{n=1}^{\infty}\left(c_{n}(f)-c_{n}\left(f_{k}\right)\right)\left(c_{n}(f)+c_{n}\left(f_{k}\right)\right)\right| \\
& \quad \leq\left(\sum_{n=1}^{\infty}\left(c_{n}(f)-c_{n}\left(f_{k}\right)\right)^{2}\right)^{1 / 2}\left(\sum_{n=1}^{\infty}\left(c_{n}(f)+c_{n}\left(f_{k}\right)\right)^{2}\right)^{1 / 2} \\
& \quad \longrightarrow 0, \text { as } k \longrightarrow \infty .
\end{aligned}
$$

This means that $\lim _{k \rightarrow \infty} \sum_{n=1}^{\infty} c_{n}^{2}\left(f_{k}\right)=\sum_{n=1}^{\infty} c_{n}^{2}(f)$.

Since $f_{k} \rightarrow f$ in $H$ as $k \rightarrow \infty, \lim _{k \rightarrow \infty}\left\|f_{k}\right\|_{H}=\|f\|_{H}$. We obtain

$$
\|f\|_{H}^{2}=\lim _{k \rightarrow \infty}\left\|f_{k}\right\|_{H}^{2}=\lim _{k \rightarrow \infty} \sum_{n=1}^{\infty} c_{n}^{2}\left(f_{k}\right)=\sum_{n=1}^{\infty} c_{n}^{2}(f) .
$$

\section{Acknowledgments}

The authors thank the reviewers for their careful reading of the paper and for the constructive comments and suggestions which have led to the improvement of the presentation of the paper. This work was supported by the National Nature Science Foundation of China (11161030) and the Specialized Research Fund for the Doctoral Program of Higher Education of China (20121501120003).

\section{References}

[1] P. B. Bailey, W. N. Everitt, and A. Zettl, "Computing eigenvalues of singular Sturm-Liouville problems," Results in Mathematics, vol. 20, no. 1-2, pp. 391-423, 1991.

[2] P. Binding, H. Langer, and M. Möller, "Oscillation results for Sturm-Liouville problems with an indefinite weight function," Journal of Computational and Applied Mathematics, vol. 171, no. 1-2, pp. 93-101, 2004.

[3] M. A. El-Gebeily, D. O’Regan, and R. Agarwal, "Characterization of self-adjoint ordinary differential operators," Mathematical and Computer Modelling, vol. 54, no. 1-2, pp. 659-672, 2011.

[4] S. Hassi, M. Möller, and H. de Snoo, "Sturm-Liouville operators and their spectral functions," Journal of Mathematical Analysis and Applications, vol. 282, no. 2, pp. 584-602, 2003.

[5] A. C. King, J. Billingham, and S. R. Otto, Differential Equations: Linear, Nonlinear, Ordinary, Partial, Cambridge University Press, Cambridge, UK, 2003.

[6] J. Sun, "On the self-adjoint extensions of symmetric ordinary differential operators with middle deficiency indices," Acta Mathematica Sinica, vol. 2, no. 2, pp. 152-167, 1986.

[7] A. Wang, J. Sun, and A. Zettl, "The classification of self-adjoint boundary conditions: separated, coupled, and mixed," Journal of Functional Analysis, vol. 255, no. 6, pp. 1554-1573, 2008.

[8] Z. Akdoğan and Z. Şaşmaz, "Sturm-Liouville problems with discontinuous potential," Bulletin of the Australian Mathematical Society, vol. 77, no. 3, pp. 485-494, 2008. 
[9] A. Wang, J. Sun, X. Hao, and S. Yao, "Completeness of eigenfunctions of Sturm-Liouville problems with transmission conditions," Methods and Applications of Analysis, vol. 16, no. 3, pp. 299-312, 2009.

[10] C.-F. Yang and X.-P. Yang, "An interior inverse problem for the Sturm-Liouville operator with discontinuous conditions," Applied Mathematics Letters, vol. 22, no. 9, pp. 1315-1319, 2009.

[11] S. Erdoğan, S. Araci, M. Acikgoz, and K. Oruçoğlu, "A class of Sturm-Liouville operators with eigenparameter dependent boundary and transmission conditions," International Journal of Pure and Applied Mathematics, vol. 84, no. 1, pp. 149-157, 2013.

[12] O. Sh. Mukhtarov, M. Kadakal, and F. Ş. Muhtarov, "Eigenvalues and normalized eigenfunctions of discontinuous SturmLiouville problem with transmission conditions," Reports on Mathematical Physics, vol. 54, no. 1, pp. 41-56, 2004.

[13] Q. Kong and Q.-R. Wang, "Using time scales to study multiinterval Sturm-Liouville problems with interface conditions," Results in Mathematics, vol. 63, no. 1-2, pp. 451-465, 2013.

[14] J. Sun and A. Wang, "Sturm-Liouville operators with interface conditions," in The Progress of Research for Math, Mech, Phys. and High Tech, vol. 12, pp. 513-516, Science Press, Beijing, China, 2008.

[15] A. Zettl, "Adjoint and self-adjoint boundary value problems with interface conditions," SIAM Journal on Applied Mathematics, vol. 16, pp. 851-859, 1968.

[16] F. S. Muhtarov and K. Aydemir, "Distributions of eigenvalues for Sturm-Liouville problem under jump conditions," Journal of New Results in Science, vol. 1, pp. 81-89, 2012.

[17] B. Chanane, "Eigenvalues of Sturm-Liouville problems with discontinuity conditions inside a finite interval," Applied Mathematics and Computation, vol. 188, no. 2, pp. 1725-1732, 2007.

[18] M. Kadakal and O. Sh. Mukhtarov, "Sturm-Liouville problems with discontinuities at two points," Computers \& Mathematics with Applications, vol. 54, no. 11-12, pp. 1367-1379, 2007.

[19] A. Zettl, Sturm-Liouville Theory, vol. 121 of Mathematical Surveys and Monographs, American Mathematical Society, Providence, RI, USA, 2005.

[20] E. C. Titchmarsh, Eigenfunction Expansions Associated with Second-Order Differential Equations, Clarendon Press, Oxford, UK, 1946.

[21] E. C. Titchmarsh, The Theory of Functions, Oxford University Press, 2nd edition, 1939.

[22] M. A. Naimark, Linear Differential Operators, Ungar, New York, NY, USA, 1968.

[23] R. A. Adams and J. J. F. Fournier, Sobolev Spaces, vol. 140 of Pure and Applied Mathematics (Amsterdam), Elsevier/Academic Press, Amsterdam, The Netherlands, 2nd edition, 2003. 


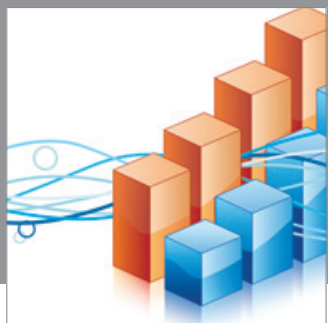

Advances in

Operations Research

mansans

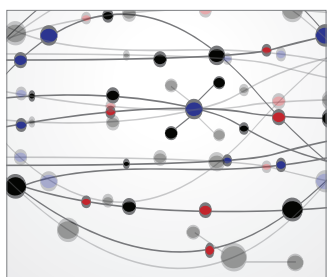

The Scientific World Journal
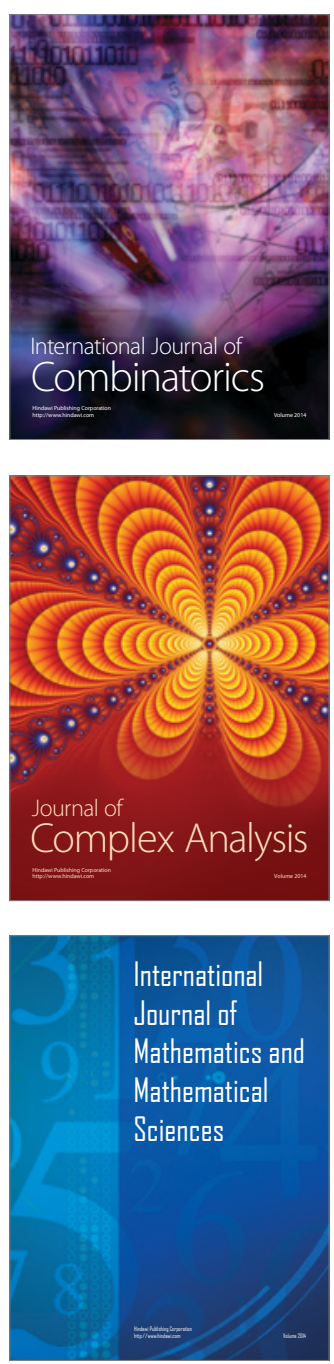
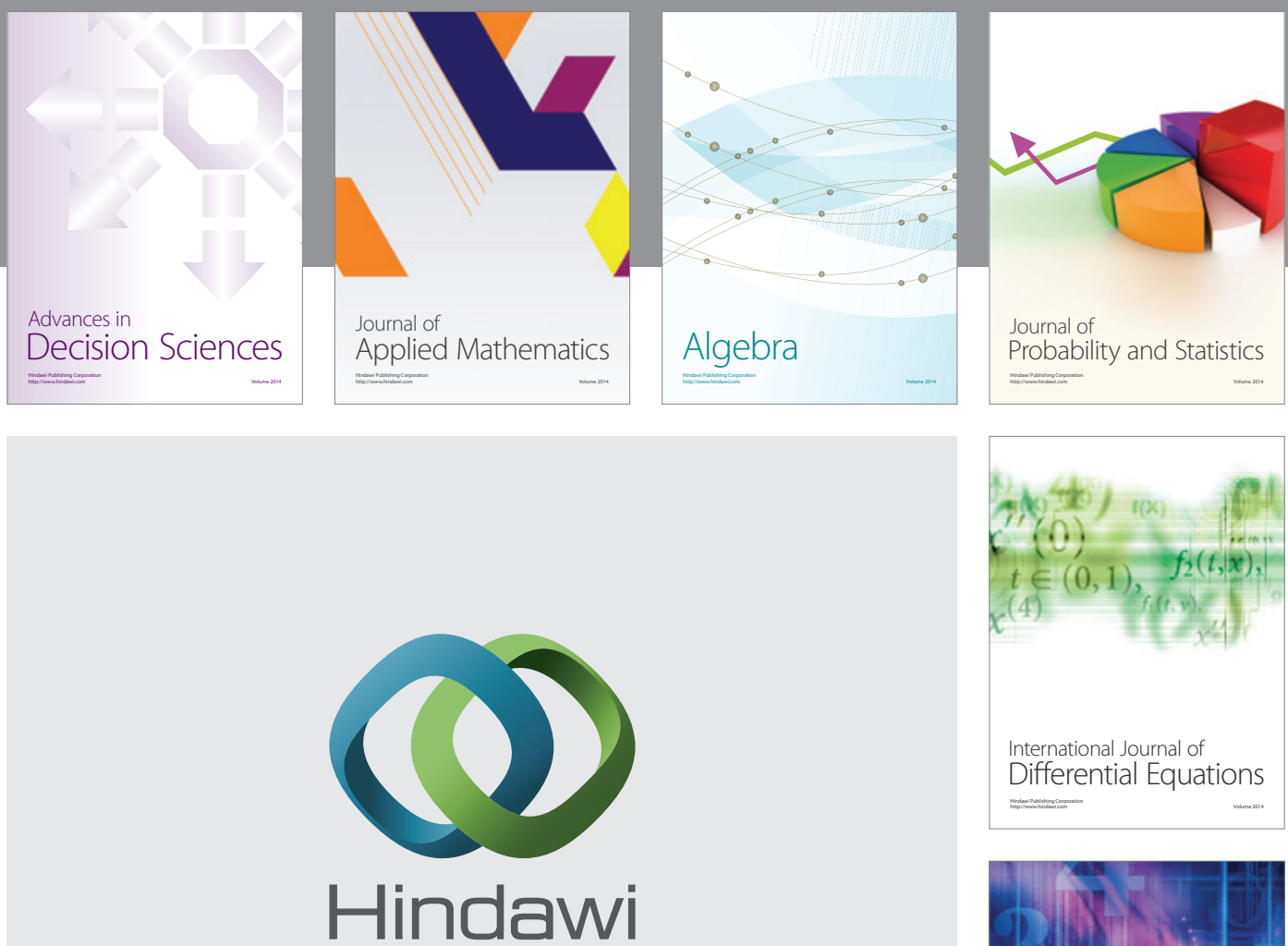

Submit your manuscripts at http://www.hindawi.com
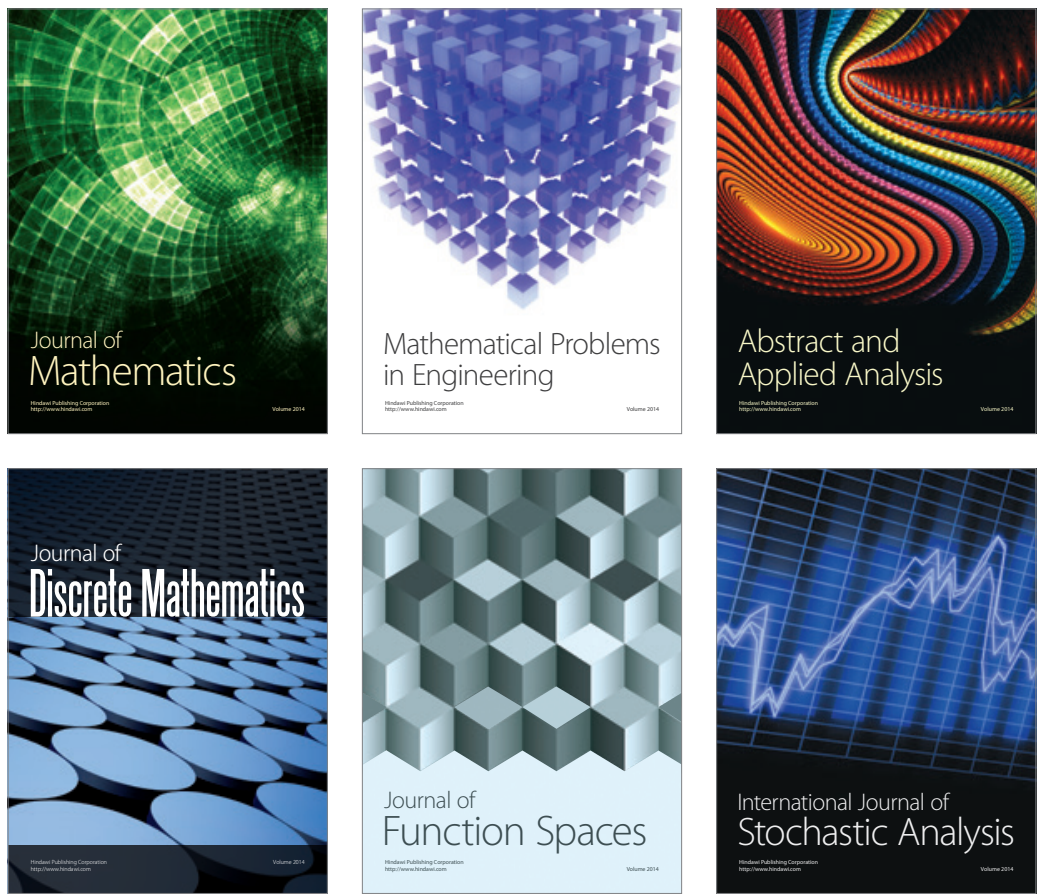

Journal of

Function Spaces

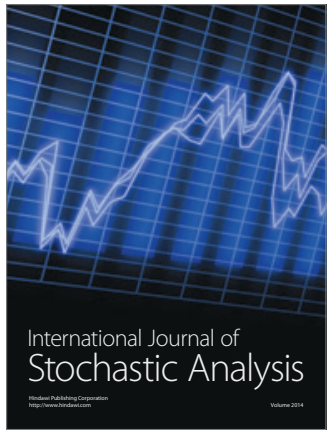

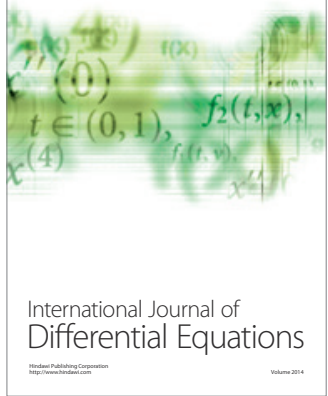
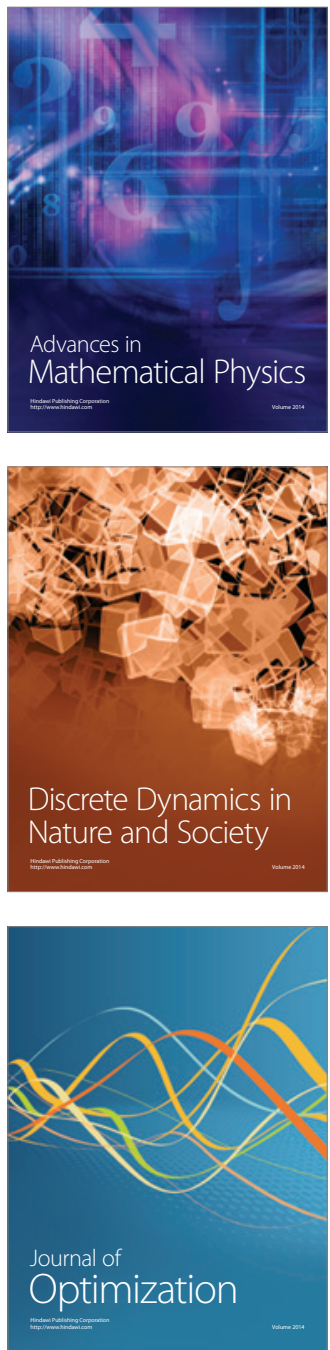